\title{
Some combinatorics of rhomboid-shaped fully packed loop configurations
}

\author{
Sabine Beill]
}

Fakultät für Mathematik, Universität Wien, Oskar-Morgenstern-Platz, 1, 1090 Wien, Austria

\begin{abstract}
The study of rhomboid-shaped fully packed loop configurations (RFPLs) is inspired by the work of Fischer and Nadeau on triangular fully packed loop configurations (TFPLs). By using the same techniques as they did some nice combinatorics for RFPLs arise. To each RFPL and to each oriented RFPL a quadruple of binary words $(\alpha, \beta ; \gamma, \delta)$ - its so-called boundary - is assigned. There are necessary conditions for the boundary of an RFPL respectively an oriented RFPL. For instance, it has to fulfill the inequality $d(\gamma)+d(\delta) \geq d(\alpha)+d(\beta)+|\alpha|_{0}|\beta|_{1}$, where $|\alpha|_{i}$ denotes the number of occurrences of $i=0,1$ in $\alpha$ and $d(\alpha)$ denotes the number of inversions of $\alpha$. Furthermore, the number of ordinary RFPLs with boundary $(\alpha, \beta ; \gamma, \delta)$ can be expressed in terms of oriented RFPLs with the same boundary. Finally, oriented RFPLs with boundary $(\alpha, \beta ; \gamma, \delta)$ such that $d(\gamma)+d(\delta)=d(\alpha)+$ $d(\beta)+|\alpha|_{0}|\beta|_{1}$ are considered. They are in bijection with rhomboid-shaped Knutson-Tao puzzles. Also, LittlewoodRichardson tableaux of defect $d$ are defined. They can be understood as a generalization of Littlewood-Richardson tableaux. Those tableaux are in bijection with rhomboid-shaped Knutson-Tao puzzles.
\end{abstract}

Résumé. L'étude des configurations de boucles compactes dans un rhomboïde ("rhomboid-shaped fully packed loop configurations", RFPLs) est inspirée des travaux de Fischer et Nadeau sur les configurations de boucles compactes dans un triangle (TFPLs). En utilisant les mêmes techniques, des résultats combinatoires sont obtenus pour les RPFLs. À chaque RPFL et à chaque RPFL orienté nous associons un quadruplet de mots binaires $(\alpha, \beta ; \gamma, \delta)$, appelé sa frontière. Il existe des conditions nécessaires pour la frontière des RPFLs, resp. des RPFLs orientés. Par exemple, la frontière $(\alpha, \beta ; \gamma, \delta)$ doit satisfaire l'inégalité $d(\gamma)+d(\delta) \geq d(\alpha)+d(\beta)+|\alpha|_{0}|\beta|_{1}$, où $|\alpha|_{i}$ désigne le nombre d'occurrences de $i=0,1$ dans $\alpha$ et $d(\alpha)$ est le nombre d'inversions de $\alpha$. D'autre part, le nombre de RPFLs ordinaires de frontière $(\alpha, \beta ; \gamma, \delta)$ est exprimé en termes de RPFLs orientés de même frontière. Enfin, nous considèrons des RPFLs orientés de frontière $(\alpha, \beta ; \gamma, \delta)$ tels que $d(\gamma)+d(\delta)=d(\alpha)+d(\beta)+|\alpha|_{0}|\beta|_{1}$. Ceux-ci sont en bijection avec les puzzles de Knutson-Tao sur un rhomboïde. De plus, nous définissons les tableaux de LittlewoodRichardson de défaut $d$, qui peuvent être vus comme des généralisations des tableaux de Littlewood-Richardson. Ces tableaux sont en bijection avec les puzzles de Knutson-Tao sur un rhomboïde.

Keywords: Fully packed loop configurations, Knutson-Tao puzzles

\footnotetext{
*Email: sabine.beil@univie.ac.at. AY0046321.

1365-8050 @ 2014 Discrete Mathematics and Theoretical Computer Science (DMTCS), Nancy, France
} 


\section{Introduction}

Fully packed loop configurations (FPLs) came up in statistical mechanics. Later, it turned out that they are in bijection with alternating sign matrices. Thus, FPLs are enumerated by the famous formula for alternating sign matrices in Zeilberger (1996). In a natural way, every FPL defines a (non-crossing) matching of the occupied external edges - the so-called link pattern $\pi$ - by matching those which are joined by a path. Crucial in the development of triangular fully packed loop configurations (TFPLs) are FPLs corresponding to a link pattern with a large number of nested arches: they admit a combinatorial decomposition in which TFPLs naturally arise. This came up in the course of the proof in Caselli et al. (2004) of a conjecture in Zuber (2004). It states that the number of FPLs corresponding to a given link pattern with $m$ nested arches is a polynomial in $m$. In the course of the study of FPLs corresponding to link patterns with nested arches some first combinatorics of TFPLs were derived, see Nadeau (2013a) and Thapper (2007). For example, necessary conditions for the existence of TFPLs were shown in Caselli et al. (2004), Fischer and Nadeau (2012) and Nadeau (2013a). One of these conditions states that $d(w) \geq d(u)+d(v)$ where $u, v, w$ are binary words that encode the boundary conditions of a TFPL and $d(u)$ denotes the number of inversions of $u$. This statement was first proven for TFPLs in Thapper (2007) and later for oriented TFPLs in Fischer and Nadeau (2012). To be more precise, for oriented TFPLs a combinatorial interpretation of the difference $d(w)-d(u)-d(v)$ is given in Fischer and Nadeau (2012). This point of view turned out fruitful: under the constraint that $d(w)-d(u)-d(v)=0$ oriented TFPLs with boundary (u,v;w) are

enumerated by the Littlewood-Richardson coefficient $c_{\lambda(u), \lambda(v)}^{\lambda(w)}$ where $\lambda(u)$ denotes the Young diagram corresponding to $u$. First, this was only shown for Dyck words $u, v, w$ in Nadeau (2013b). Later, it was extended to all ordinary and to oriented TFPLs with boundary $(u, v ; w)$ in Fischer and Nadeau (2012). More precisely, a bijection between oriented TFPLs with boundary $(u, v ; w)$ and Knutson-Tao puzzles with boundary $(u, v ; w)$ which are enumerated by Littlewood-Richardson coefficients was constructed. The goal of this article is to develop an analogous theory for RFPLs by using the techniques introduced for TFPLs in Fischer and Nadeau (2012).

\section{Definitions}

\subsection{Words}

Throughout this article when it is spoken of a word $\omega$ of length $N$ it is referred to a finite sequence $\omega=\omega_{1} \omega_{2} \cdots \omega_{N}$ where $\omega_{i} \in\{0,1\}$ for all $1 \leq i \leq N$. In context with RFPLs words play an important role. For that reason, some notations are introduced a priori. Given a word $\omega$ the number of occurrences of 0 (resp. 1) in $\omega$ is denoted by $|\omega|_{0}$ (resp. $|\omega|_{1}$ ). Furthermore, it is said that two words $\omega, \sigma$ of length $N$ satisfy $\omega \leq \sigma$ if $\left|\omega_{1} \cdots \omega_{n}\right|_{1} \leq\left|\sigma_{1} \cdots \sigma_{n}\right|_{1}$ holds for all $1 \leq n \leq N$. The number of inversions of $\omega$, i.e. of pairs $(i, j)$ where $1 \leq i<j \leq N$ that satisfy $\omega_{i}=1$ and $\omega_{j}=0$, is denoted by $d(\omega)$.

It is a well known fact that words are in bijection with Young diagrams. Throughout this article the following bijection is chosen: given a word $\omega$ a path on the square lattice is constructed by drawing a $(-1,0)$-step if $\omega_{i}=0$ and a $(0,-1)$-step if $\omega_{i}=1$ for i from 1 to $N$. Additionally, to the left of the path's starting point and up its ending point lines are drawn. The resulting region then encloses the wanted Young diagram which is denoted by $\lambda(\omega)$. In Figure 1 an example of the previous bijection is given. Due to the choice of the bijection the number of columns (resp. rows) of $\lambda(\omega)$ equals $|\omega|_{0}$ (resp. $|\omega|_{1}$ ). Furthermore, for two words $\omega$ and $\sigma$ of length $N$ it holds $\omega \leq \sigma$ if and only if $\lambda(\omega)$ is contained in $\lambda(\sigma)$ and the number of cells of $\lambda(\omega)$ is given by $d(\omega)$. 


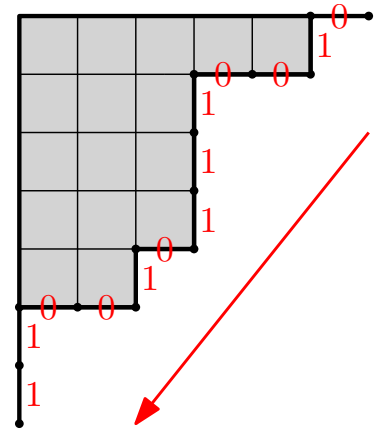

Fig. 1: The bijection $\lambda$ between words and Young diagrams.

There is one more notation in connection with words that will be needed. Given a binary word $\omega$ of length $N$ the number of zeroes in $\omega$ between the $(j-1)$-st and the $j$-th one in $\omega$, where the ones are counted from left to right, is denoted by $|\omega|_{0, j}$ for $2 \leq j \leq|\omega|_{1}$. In addition, the number of zeroes after the last one in $\omega$ is denoted by $|\omega|_{0,|\omega|_{1}+1}$.

\subsection{Rhomboid-shaped fully packed loop configurations}

In this subsection ordinary and oriented rhomboid-shaped fully packed loop configurations (RFPLs) are defined. Furthermore, to each ordinary and to each oriented RFPL a boundary is assigned. Also, an expression of the number of ordinary RFPLs with a given boundary in terms of oriented RFPLs with the same boundary is given. At the end of this subsection, necessary conditions for the existence of RFPLs and oriented RFPLs are formulated.

Definition 1 Let $M$ and $N$ be positive integers such that $M \geq N$. The graph $R^{M, N}$ is defined as the induced subgraph of the square grid induced by the vertex set $\mathcal{T}_{t} \cup \mathcal{P} \cup \mathcal{T}_{b}$ where

1. $\mathcal{T}_{t}=\{(M-N+i+j, M-N+i): 0 \leq i \leq N-1,0 \leq j \leq 2(N-i)\}$,

2. $\mathcal{P}=\{(i+j, i): 0 \leq i \leq M-N-1,0 \leq j \leq 2 N+1\}$ and

3. $\mathcal{T}_{b}=\{(i+j-1,-i): 1 \leq i \leq N, 0 \leq j \leq 2(-i+N+1)\}$.

The vertices of $R^{M, N}$ are partitioned into odd and even vertices in a chessboard manner such that the leftmost vertex of the top row of $R^{M, N}$ is odd. In the pictures, odd vertices are illustrated by disks and even vertices by squares. The following vertices of $R^{M, N}$ play an important role: the leftmost vertices $\mathcal{L}_{o}^{M}=\left\{L_{o, 1}, \ldots, L_{o, M}\right\}$ of each of the top $M$ rows of $R^{M, N}$, the rightmost vertices $\mathcal{R}_{o}^{N}=$ $\left\{R_{o, 1}, \ldots, R_{o, N}\right\}$ of each of the top $N$ rows of $R^{M, N}$, the leftmost vertices $\mathcal{L}_{e}^{N}=\left\{L_{e, 1}, \ldots, L_{e, N}\right\}$ of each of the bottom $N$ rows of $R^{M, N}$ and the rightmost vertices $\mathcal{R}_{e}^{M}=\left\{R_{e, 1}, \ldots, R_{e, N}\right\}$ of each of the bottom $M$ rows of $R^{M, N}$. All vertices are numbered from left to right.

Definition 2 Let $M \geq N$ be positive integers.

1. An oriented rhomboid-shaped fully packed loop configuration of size $(M, N)$ is an induced subgraph $f$ of $R^{M, N}$ together with an orientation of the edges such that: 
(a) All vertices of $\mathcal{L}_{e}^{N} \cup \mathcal{L}_{o}^{N}$ are either of degree 0 or incident to precisely one outgoing edge.

(b) All vertices of $\mathcal{R}_{e}^{N} \cup \mathcal{R}_{o}^{N}$ are either of degree 0 or incident to precisely one incoming edge.

(c) All other vertices of $R^{M, N}$ have degree 2 and are incident to an incoming and an outgoing edge.

2. Let $\alpha, \gamma$ be words of length $M$ and $\beta, \delta$ words of length $N$. An oriented RFPL $f$ of size $(M, N)$ has boundary $(\alpha, \beta ; \gamma, \delta)$ if for each $1 \leq i \leq M$ and for each $1 \leq j \leq N$ the following holds:

(a) $\alpha_{i}=1$ if and only if $L_{o, i}$ has degree 1 ;

(b) $\beta_{j}=0$ if and only if $R_{o, j}$ has degree 1 ;

(c) $\gamma_{i}=1$ if and only if $R_{e, i}$ has degree 1 ;

(d) $\delta_{j}=0$ if and only if $L_{e, j}$ has degree 1.

The set of oriented RFPLs with boundary $(\alpha, \beta ; \gamma, \delta)$ is denoted by $\vec{R}_{\alpha, \beta}^{\gamma, \delta}$ and its cardinality by $\vec{r}_{\alpha, \beta}^{\gamma, \delta}$.

In Figure 2 an example of an oriented RFPL is depicted. By conditions (a) and (b) in the definition of

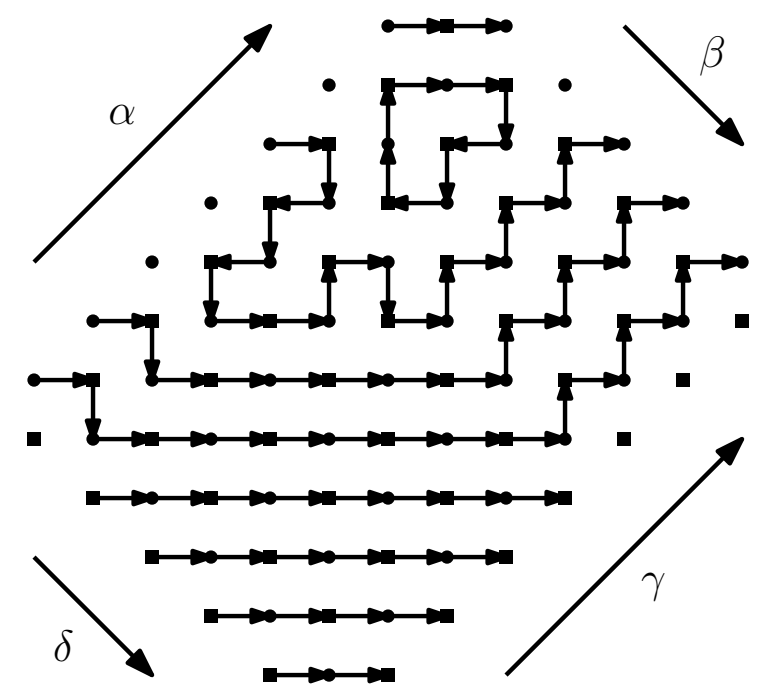

Fig. 2: An oriented RFPL of size (7,5) with boundary (1100101, 01000; 1111000, 10000).

oriented RFPLs it follows that in an oriented RFPL a path joins neither two vertices in $\mathcal{L}_{e}^{N} \cup \mathcal{L}_{o}^{N}$ nor two vertices in $\mathcal{R}_{e}^{N} \cup \mathcal{R}_{o}^{N}$. Moreover, the orientation of each path that connects a vertex in $\mathcal{L}_{e}^{N} \cup \mathcal{L}_{o}^{N}$ with a vertex in $\mathcal{R}_{e}^{N} \cup \mathcal{R}_{o}^{N}$ is uniquely determined. Hence, oriented RFPLs where by convention all closed paths are oriented clockwise are said to be (ordinary) RFPLs. The set of RFPLs with boundary $(\alpha, \beta ; \gamma, \delta)$ is denoted by $R_{\alpha, \beta}^{\gamma, \delta}$ and its cardinality by $r_{\alpha, \beta}^{\gamma, \delta}$. Then the number of RFPLs can be expressed in terms of oriented RFPLs in an analogous way as the number of TFPLs is expressed in terms of oriented TFPLs in 
Fischer and Nadeau (2012). For that purpose, the following weighted enumeration of oriented RFPLs is needed:

$$
\vec{r}_{\alpha, \beta}^{\gamma, \delta}(q)=\sum_{f \in \vec{R}_{\alpha, \beta}^{\gamma, \delta}} q^{N} \circlearrowleft_{(f)-N} \circlearrowright(f)
$$

where $N^{\circlearrowleft}(f)$ denotes the number of closed paths in $f$ that are oriented counterclockwise and $N^{\circlearrowright}(f)$ the number of closed paths in $f$ that are oriented clockwise.

Proposition 1 Let $\rho$ be a primitive sixth root of unity so that $\rho$ satisfies $\rho+1 / \rho=1$. Then

$$
r_{\alpha, \beta}^{\gamma, \delta}=\vec{r}_{\alpha, \beta}^{\gamma, \delta}(\rho)
$$

Finally, necessary conditions for the existence of oriented RFPLs are given. They are similar to those obtained for TFPLs in Caselli et al. (2004), Thapper (2007), Nadeau (2013a) and Fischer and Nadeau (2012).

Theorem 1 Let $M \geq N$ be two positive integers, $\alpha, \gamma$ be words of length $M$ and $\beta, \delta$ words of length $N$ such that $\vec{r}_{\alpha, \beta}^{\gamma, \delta}>0$. Then the following holds:

1. $|\alpha|_{0}=|\gamma|_{0}$ and $|\beta|_{0}=|\delta|_{0}$,

2. $\alpha \leq \gamma$ and $\beta \leq \delta$,

3. $d(\gamma)+d(\delta) \geq d(\alpha)+d(\beta)+|\alpha|_{0}|\beta|_{1}$.

Since $r_{\alpha, \beta}^{\gamma, \delta} \leq \vec{r}_{\alpha, \beta}^{\gamma, \delta}$ one immediately gets:

Corollary 1 The conclusions of Theorem 1 also hold if $r_{\alpha, \beta}^{\gamma, \delta}>0$.

The boundary of the oriented RFPL depicted in Figure 2 is $(1100101,01000 ; 1111000,10000)$. It can easily be checked that it satisfies all conditions in Theorem 1

\section{Perfect matchings and Path tangles}

In this section, the techniques used in Fischer and Nadeau (2012) to prove for instance necessary conditions for the existence of oriented TFPLs are adapted to oriented RFPLs. More precisely, oriented RFPLs are decomposed into two perfect matchings which are disjoint as subsets of the edges of $R^{M, N}$. Each of these two matchings then is in bijection with certain non-intersecting lattice paths. This approach is crucial for the proof of Theorem 1 given in this article.

\subsection{Perfect matchings}

For $\alpha, \gamma$ two words of length $M$, the graph $R_{o}^{M}(\alpha, \gamma)$ is defined as the induced subgraph of $R^{M, N}$ that is obtained by removing all vertices of $\mathcal{R}_{o}^{N} \cup \mathcal{L}_{e}^{N}$, all those vertices $L_{o, i} \in \mathcal{L}_{o}^{M}$ such that $\alpha_{i}=0$ and all those vertices $R_{e, i} \in \mathcal{R}_{e}^{M}$ such that $\gamma_{i}=0$. In the following, perfect matchings on $R_{o}^{M}(\alpha, \gamma)$ are regarded. Given such a perfect matching $M_{o}$ by orienting each of its edges from its odd to its even vertex one gets edges oriented in the directions up, down, left or right. The associated sets of edges are denoted by $\mathcal{O}_{U}\left(M_{o}\right), \mathcal{O}_{D}\left(M_{o}\right), \mathcal{O}_{L}\left(M_{o}\right)$ and $\mathcal{O}_{R}\left(M_{o}\right)$ and its cardinalities by $o_{U}\left(M_{o}\right), o_{D}\left(M_{o}\right), o_{L}\left(M_{o}\right)$ and 
$o_{R}\left(M_{o}\right)$. Similarly, the graph $R_{e}^{N}(\beta, \delta)$ for two words $\beta, \delta$ of length $N$ is defined as the induced subgraph of $R^{M, N}$ that is obtained by removing all vertices of $\mathcal{L}_{o}^{M} \cup \mathcal{R}_{e}^{M}$, all those vertices $R_{o, i} \in \mathcal{R}_{o}^{N}$ such that $\beta_{i}=1$ and all those vertices $L_{e, i} \in \mathcal{L}_{e}^{N}$ such that $\delta_{i}=1$. Furthermore, given a perfect matching $M_{e}$ on $R_{e}^{N}(\beta, \delta)$ by orienting each of its edges from its even to its odd vertex one obtains edges oriented in the directions up, down, left or right. The associated sets of edges are denoted by $\mathcal{E}_{U}\left(M_{e}\right), \mathcal{E}_{D}\left(M_{e}\right), \mathcal{E}_{L}\left(M_{e}\right)$ and $\mathcal{E}_{R}\left(M_{e}\right)$ and its cardinalities by $\varepsilon_{U}\left(M_{e}\right), \varepsilon_{D}\left(M_{e}\right), \varepsilon_{L}\left(M_{e}\right)$ and $\varepsilon_{R}\left(M_{e}\right)$.

The introduction of these graphs and perfect matchings is motivated by the following result:

Proposition 2 Let $M \geq N$ be two positive integers and $\alpha, \gamma$ be words of length $M$ and $\beta, \delta$ be words length $N$. For any oriented RFPL $r$ of size $(M, N)$ with boundary $(\alpha, \beta ; \gamma, \delta)$ denote by $M_{o}(r)$ (resp. $M_{e}(r)$ ) the subset of its edges which are oriented from an odd to an even vertex (resp. from an even to an odd vertex). Then the map

$$
r \mapsto\left(M_{o}(r), M_{e}(r)\right)
$$

is a bijection from $\vec{R}_{\alpha, \gamma}^{\gamma, \delta}$ to the set of ordered pairs $\left(M_{o}, M_{e}\right)$, where $M_{o}$ is a perfect matching on $R_{o}^{M}(\alpha, \beta)$ and $M_{e}$ is a perfect matching on $R_{e}^{N}(\beta, \delta)$ such that $M_{o}$ and $M_{e}$ are disjoint as subsets of edges of $R^{M, N}$.

The previous proposition provides a proof of the first two conditions of Theorem 1 Certain edges of a perfect matching on $R_{o}^{M}(\alpha, \gamma)$ or $R_{e}^{N}(\beta, \delta)$ can be enumerated solely in terms of $M, N, \alpha, \beta, \gamma$ and $\delta$ :

Proposition 3 Let $M \geq N$ be positive integers, let $\alpha, \gamma$ be words of length $M$ such that $|\alpha|_{1}=|\gamma|_{1}$ and let $\beta, \delta$ be words of length $N$ such that $|\beta|_{0}=|\delta|_{0}$.

1. For any perfect matching $M_{o}$ on $R_{o}^{M}(\alpha, \gamma)$ the following identities hold:

(a) $o_{U}\left(M_{o}\right)+o_{D}\left(M_{o}\right)+o_{L}\left(M_{o}\right)+o_{R}\left(M_{o}\right)=M N+|\alpha|_{1}$;

(b) $o_{L}\left(M_{o}\right)+o_{D}\left(M_{o}\right)=d(\gamma)-d(\alpha)$.

2. For any perfect matching $M_{e}$ on $R_{e}^{N}(\beta, \delta)$ the following identities hold:
(a) $\varepsilon_{U}\left(M_{e}\right)+\varepsilon_{D}\left(M_{e}\right)+\varepsilon_{L}\left(M_{e}\right)+\varepsilon_{R}\left(M_{e}\right)=M N+|\beta|_{0} ;$
(b) $\varepsilon_{L}\left(M_{e}\right)+\varepsilon_{U}\left(M_{e}\right)=d(\delta)-d(\beta)$.

\subsection{Path tangles}

In the following, it is shown that perfect matchings on $R_{o}^{M}(\alpha, \gamma)$ where $|\alpha|_{1}=|\gamma|_{1}$ and perfect matchings on $R_{e}^{N}(\beta, \delta)$ where $|\beta|_{0}=|\delta|_{0}$ are in bijection with certain non-intersecting lattice paths. Hence, let $\alpha, \gamma$ be words of length $M$ such that $|\alpha|_{1}=|\gamma|_{1}$ and $\beta, \delta$ be words of length $N$ such that $|\beta|_{0}=|\delta|_{0}$. Next, the bijections between perfect matchings on $R_{o}^{M}(\alpha, \gamma)$ and certain configurations of blue paths and between perfect matchings on $R_{e}^{N}(\beta, \delta)$ and certain configurations of red paths are described. As a start, perfect matchings on $R_{o}^{M}(\alpha, \gamma)$ are considered. At first, a new set of blue vertices is added to $R^{M, N}$ as follows: in the middle of each horizontal edge of $R^{M, N}$ which has an odd vertex to its left a blue vertex is added. Furthermore, given a perfect matching $M_{o}$ on $R_{o}^{M}(\alpha, \gamma)$ blue oriented edges are added as indicated in Figure 3 In that way, non-intersecting blue lattice paths are obtained. To be more precise, it is set $I_{\gamma}=\left\{1 \leq i_{1}<\cdots<i_{|\gamma|_{0}} \leq M\right\}$ the set of indices $i$ such that $\gamma_{i}=0$ and similarly $I_{\alpha}=\left\{1 \leq j_{1}<\cdots<j_{|\alpha|_{0}} \leq M\right\}$ the set of indices $j$ such that $\alpha_{j}=0$. Furthermore, for all 

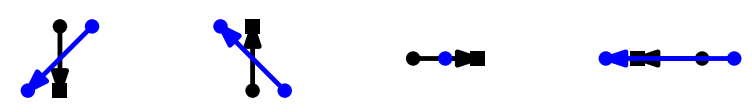

Fig. 3: From perfect matchings of $R_{o}^{M}(\alpha, \gamma)$ to nonintersecting blue lattice paths.

$1 \leq m, n \leq|\alpha|_{0}$ the point $\left(i_{m}-\frac{1}{2}+N, i_{m}-N-1\right)$ is denoted by $D_{m}$, the point $\left(j_{n}-\frac{1}{2}, j_{n}-1\right)$ is denoted by $E_{n}$ and the set of all paths from $D_{m}$ to $E_{n}$ using steps $(-1,1),(-1,-1),(-2,0)$ is denoted by $\mathcal{P}\left(D_{m}, E_{n}\right)$.

Proposition 4 The correspondence defined above is a bijection between:

1. Perfect matchings on $R_{o}^{M}(\alpha, \gamma)$, and

2. Non-intersecting paths $\left(P_{1}, \ldots, P_{|\alpha|_{0}}\right)$ where $P_{m} \in \mathcal{P}\left(D_{m}, E_{m}\right)$.

The set of non-intersecting paths $\left(P_{1}, \ldots, P_{|\alpha|_{0}}\right)$ where $P_{m} \in \mathcal{P}\left(D_{m}, E_{m}\right)$ is denoted by $\mathcal{P}(\alpha, \gamma)$.

Next, perfect matchings on $R_{e}^{N}(\beta, \delta)$ are considered. As a start, a new set of red vertices is added to $R^{M, N}$ as follows: in the middle of each horizontal edge of $R^{M, N}$ which has an odd vertex to its right a red vertex is added. Then to a given perfect matching $M_{e}$ of $R_{e}^{N}(\beta, \delta)$ red oriented edges are added as indicated in Figure 4 . In that way, red non-intersecting lattice paths are obtained. More pre-

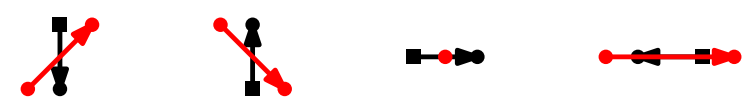

Fig. 4: From perfect matchings on $R_{e}^{N}(\beta, \delta)$ to non-intersecting red lattice paths.

cisely, it is set $I_{\delta}^{\prime}=\left\{1 \leq i_{1}^{\prime}<\cdots<i_{|\delta|_{1}}^{\prime} \leq N\right\}$ the set of indices $i^{\prime}$ such that $\delta_{i^{\prime}}=1$ and similarly $I_{\beta}^{\prime}=\left\{1 \leq j_{1}^{\prime}<\cdots<j_{|\beta|_{1}}^{\prime} \leq N\right\}$ the set of all indices $j^{\prime}$ such that $\beta_{j^{\prime}}=1$. Now, we define $D_{m}^{\prime}$ as the point $\left(i_{m}^{\prime}-\frac{1}{2},-i_{m}^{\prime}\right), E_{n}^{\prime}$ as the point $\left(M-\frac{1}{2}+j_{n}^{\prime}, M-j_{n}^{\prime}\right)$ and $\mathcal{P}^{\prime}\left(D_{m}^{\prime}, E_{n}^{\prime}\right)$ as the set of all paths from $D_{m}^{\prime}$ to $E_{n}^{\prime}$ using steps $(1,1),(1,-1),(2,0)$ for all $1 \leq m, n \leq|\beta|_{1}$.

Proposition 5 The correspondence defined above is a bijection between:

1. Perfect matchings on $R_{e}^{N}(\beta, \delta)$, and

2. Non-intersecting paths $\left(P_{1}^{\prime}, \ldots, P_{|\beta|_{1}}^{\prime}\right)$ where $P_{m}^{\prime} \in \mathcal{P}^{\prime}\left(D_{m}^{\prime}, E_{m}^{\prime}\right)$.

The set of non-intersecting paths $\left(P_{1}^{\prime}, \ldots, P_{|\beta|_{1}}^{\prime}\right)$ where $P_{m}^{\prime} \in \mathcal{P}^{\prime}\left(D_{m}^{\prime}, E_{m}^{\prime}\right)$ is denoted by $\mathcal{P}^{\prime}(\beta, \delta)$.

By Proposition 2 a perfect matching $M_{o}$ on $R_{o}^{M}(\alpha, \gamma)$ and a perfect matching $M_{e}$ on $R_{e}^{N}(\beta, \delta)$ give rise to an oriented RFPL if they are disjoint as subsets of the edges of $R^{M, N}$. That constraint leads to the following bijection:

Proposition 6 The set $\vec{R}_{\alpha, \beta}^{\gamma, \delta}$ is in bijection with the set of pairs $(B, R) \in \mathcal{P}(\alpha, \gamma) \times \mathcal{P}^{\prime}(\beta, \delta)$ that fulfill the following two conditions:

1. No diagonal step of $B$ can cross a diagonal step of $R$. 
2. Each middle point of a blue (resp. red) horizontal step is used by a red (resp. blue) step.

Such configurations are said to be (blue-red) path tangles (with boundary $(\alpha, \beta ; \gamma, \delta))$ and the set of path tangles with boundary $(\alpha, \beta ; \gamma, \delta)$ is denoted by $\operatorname{BlueRed}(\alpha, \beta ; \gamma, \delta)$.

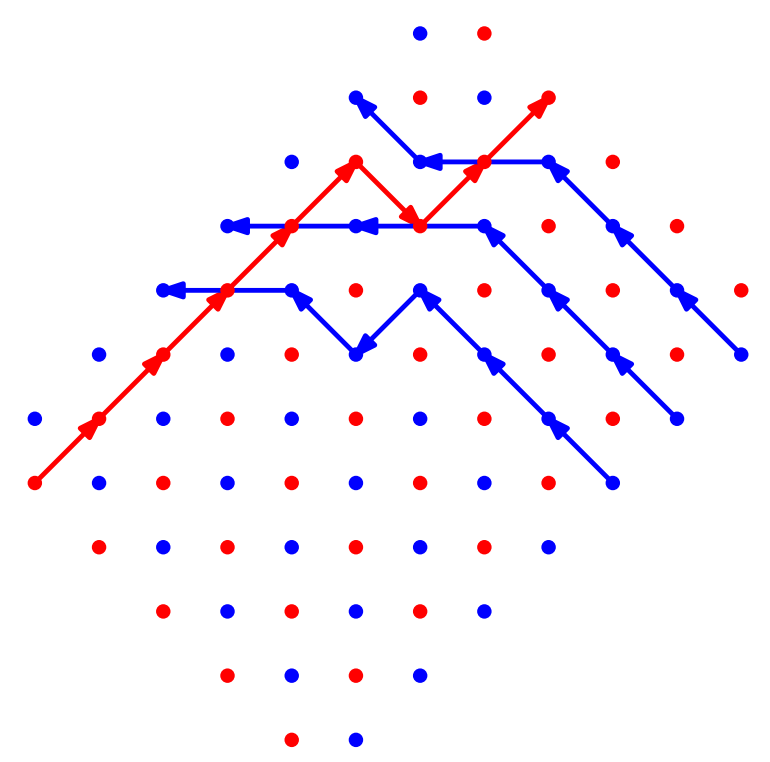

Fig. 5: The path tangle corresponding to the oriented RFPL in Figure 2

\section{Configurations of excess 0}

The third condition of Theorem 11 can be rephrased as $d(\gamma)+d(\delta)-d(\alpha)-d(\beta)-|\alpha|_{0}|\beta|_{1} \geq 0$.

Definition 3 The integer $d(\gamma)+d(\delta)-d(\alpha)-d(\beta)-|\alpha|_{0}|\beta|_{1}$ is said to be the excess of $(\alpha, \beta ; \gamma, \delta)$ and is denoted by $\operatorname{exc}(\alpha, \beta ; \gamma, \delta)$.

The excess of the boundary $(\alpha, \beta ; \gamma, \delta)$ of an oriented RFPL turns out to have the following combinatorial interpretation:

Theorem 2 Let $f$ be an oriented RFPL with boundary $(\alpha, \beta ; \gamma, \delta)$. Then

$$
\operatorname{exc}(\alpha, \beta ; \gamma, \delta)=I+I+m \longrightarrow+\infty+\infty+
$$

Here, for example denotes the number of occurrences of the very local configuration in $f$.

The third condition in Theorem 1 is an immediate consequence of Theorem 2 For example, the boundary of the oriented RFPL depicted in Figure 2 is of excess 3. On the other hand, the combinatorial interpretation of the excess suggests to regard cases of small excess. Here, only the case when the excess is zero 
is treated. The following characterization of oriented RFPLs with boundary of excess 0 is an immediate consequence of Theorem 2 .

Corollary 2 Let $f$ be an oriented RFPL with boundary $(\alpha, \beta ; \gamma, \delta)$. Then exc $(\alpha, \beta ; \gamma, \delta)=0$ if and only if none of the following configurations occurs in $f$ :

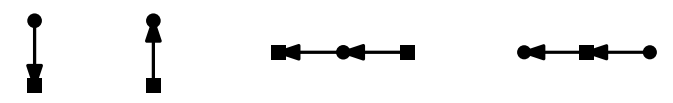

In Fischer and Nadeau (2012) a bijection between oriented TFPLs of excess 0 and Knutson-Tao puzzles is given. That bijection can be extended to a bijection between oriented RFPLs of excess 0 and rhomboidshaped Knutson-Tao puzzles.

Definition 4 (Knutson and Tao (2003)) A puzzle piece is defined as one of the following equilateral plane figures with side length 1 and labelled edges:

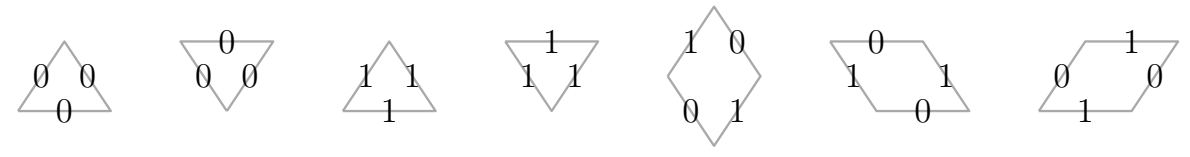

A decomposition $P$ of the rhomboid with vertices $(0,0),\left(\frac{M}{2}, \frac{M \sqrt{3}}{2}\right),\left(\frac{M+N}{2}, \frac{(M-N) \sqrt{3}}{2}\right)$ and $\left(\frac{N}{2},-\frac{N \sqrt{3}}{2}\right)$ into unit triangles and unit rhombi, all edges labelled 0 or 1 , such that each region is a puzzle piece is said to be a rhomboid-shaped Knutson-Tao puzzle of size $(M, N)$. A quadruple $(\alpha, \beta ; \gamma, \delta)$ is said to be the boundary of a rhomboid-shaped Knutson-Tao-puzzle $P$ if $\alpha$ equals the binary word that is obtained by reading the labels along the leftmost NE-SW diagonal of $P$ from left to right, $\beta$ equals the word that is obtained by reading the labels along the rightmost NW-SE diagonal of $P$ from left to right, $\gamma$ equals the word that is obtained by reading the labels along the rightmost NE-SW diagonal of $P$ from left to right and $\delta$ the binary word that is obtained by reading the labels along the leftmost NW-SE diagonal of $P$ from left to right.

Proposition 7 Let $\alpha, \gamma$ be words of length $M$ and $\beta, \delta$ be words of length $N$ such that exc $(\alpha, \beta ; \gamma, \delta)=0$. Then the set $\vec{R}_{\alpha, \beta}^{\gamma, \delta}$ is in bijection with the set of rhomboid-shaped Knutson-Tao puzzles with boundary $(\alpha, \beta ; \gamma, \delta)$.

An example of an oriented RFPL of excess 0 and its corresponding rhomboid-shaped Knutson-Tao-puzzle is given in Figure 6. In Vakil (2003) Tao's "proof without words" bijection between Knutson-Tao puzzles of triangular shape and Littlewood-Richardson tableaux is introduced. Its extension to rhomboid-shaped Knutson-Tao puzzles leads to a bijection between rhomboid-shaped Knutson-Tao puzzles and the following generalization of Littlewood-Richardson tableaux:

Definition 5 Let $d=\left(d_{i}\right)_{i=1}^{k}$ be a $k$-tuple of non-negative integers.

1. A lattice permutation of defect $d$ is a sequence $a_{1} \cdots a_{n}$ where $a_{j} \in\{1, \ldots, k+1\}$ such that for each $1 \leq m \leq n$ and for each $1 \leq i \leq k$ the following inequality holds:

$$
\left|\left\{1 \leq j \leq m: a_{j}=i\right\}\right| \geq\left|\left\{1 \leq j \leq m: a_{j}=i+1\right\}\right|-d_{i}
$$

2. A semi-standard Young tableau of skew shape whose reverse reading word is a lattice permutation with defect $d$ is said to be a Littlewood-Richardson tableau of defect $d$. 

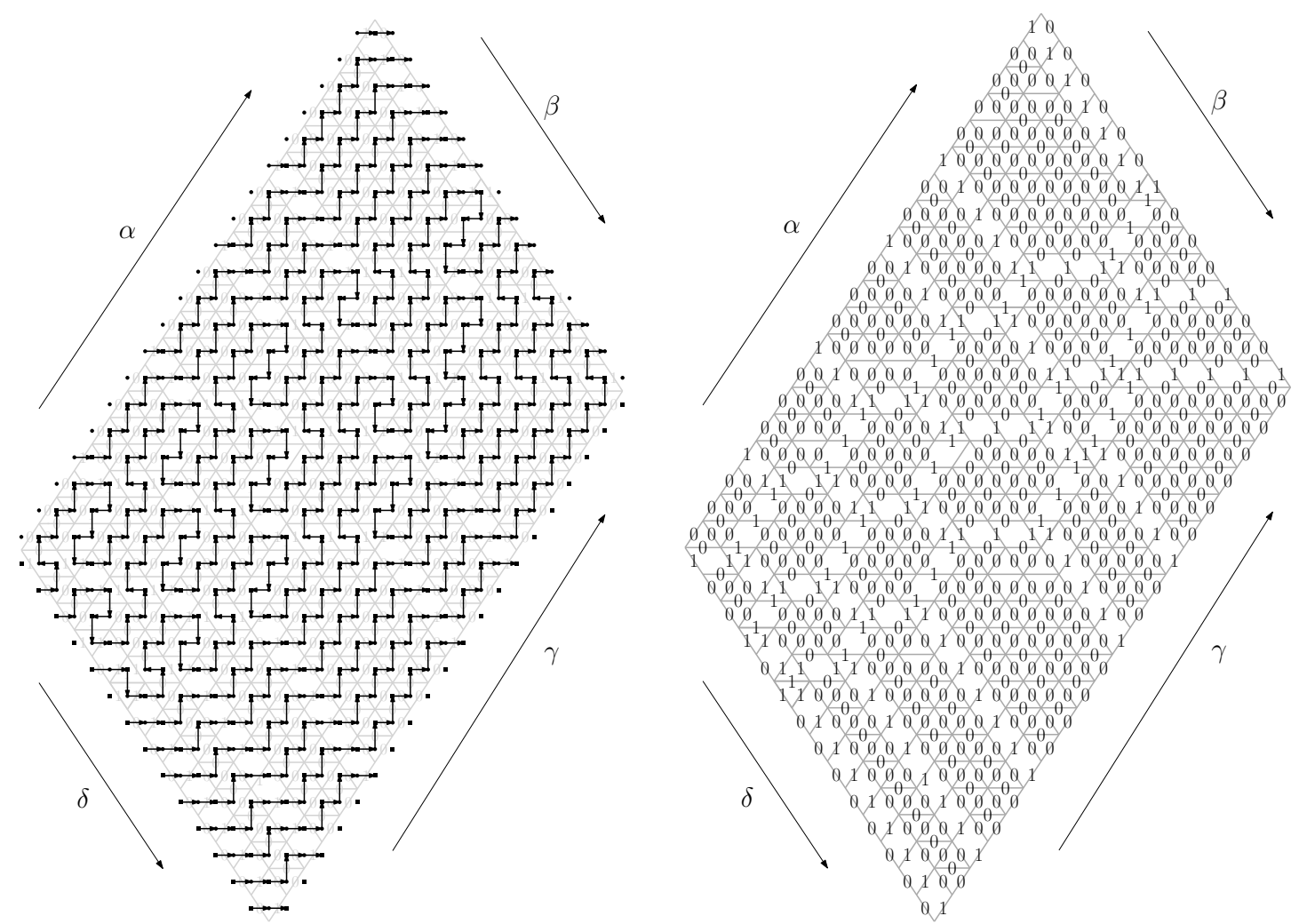

Fig. 6: An oriented RFPL and its corresponding rhomboid-shaped Knutson-Tao puzzle of size $(20,14)$ with boundary (00010001000100100001, 00000010001001; 10100100001001000000, 10010100000000).

Given a rhomboid-shaped Knutson-Tao puzzle its boundary $(\alpha, \beta ; \gamma, \delta)$ has to fulfill the following inequalities:

$$
|\alpha|_{0} \geq \sum_{k=j+1}^{|\beta|_{1}+1}\left(|\delta|_{0, k}-|\beta|_{0, k}\right)
$$

for all $1 \leq j \leq|\beta|_{1}$.

Theorem 3 Let $M \geq N$ be positive integers. Furthermore, let $\alpha, \gamma$ be words of length $M$ and $\beta, \delta$ be words of length $N$ such that the conditions of Theorem 1$] \operatorname{exc}(\alpha, \beta ; \gamma, \delta)=0$ and (6) are satisfied. Then $\vec{r}_{\alpha, \beta}^{\gamma, \delta}$ equals the number of Littlewood-Richardson tableaux of defect $\left(|\delta|_{0, i}\right)_{i=2}^{|\delta|_{1}}$ that are of skew shape $\lambda(\gamma) / \lambda(\alpha)$ and of weight $\left(|\alpha|_{0}-\sum_{k=j+1}^{|\beta|_{1}+1}\left(|\delta|_{0, k}-|\beta|_{0, k}\right)\right)_{j=1}^{|\beta|_{1}}$. 
Note, that $\sum_{j=1}^{|\beta|_{1}}\left(|\alpha|_{0}-\sum_{k=j+1}^{|\beta|_{1}+1}\left(|\delta|_{0, k}-|\beta|_{0, k}\right)\right)=|\alpha|_{0}|\beta|_{1}-d(\delta)+d(\beta)=d(\gamma)-d(\alpha)$. The latter equals the number of cells of the Young diagram of skew shape $\lambda(\gamma) / \lambda(\alpha)$. In Figure 7 , an example for the bijection between rhomboid-shaped Knutson-Tao puzzles and Littlewood-Richarson tableaux of defect $d$ is depicted. In the picture, the puzzle pieces of the rhomboid-shaped Knutson-Tao puzzle that are triangles with all edges labelled 1 are coloured black and all labels are deleted. In Knutson and Tao
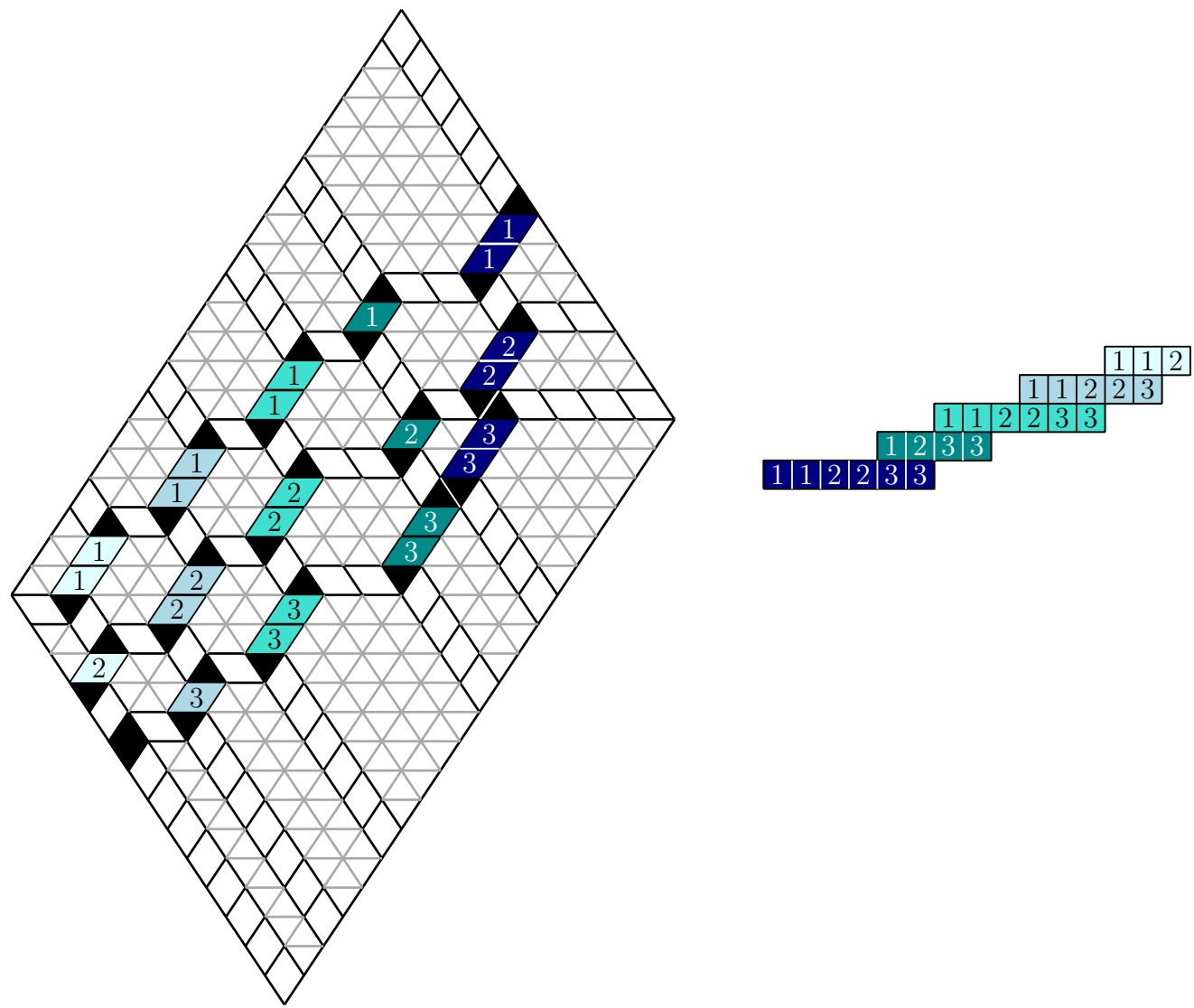

Fig. 7: The rhomboid-shaped Knutson-Tao puzzle depicted in Figure 6 and its image under the extension of Tao's "proof without words" bijection, which is a Littlewood-Richardson tableau of defect $(2,1)$ and of weight $(9,8,7)$.

(2003) the following corollary of Theorem 3 is proven for the case when $M=N$ and $\alpha, \beta, \gamma$ are words of length $N$ such that $|\alpha|_{1}=|\beta|_{1}=|\gamma|_{1}$. To state it one further notation is needed. Given a Young diagram $\lambda=\left(\lambda_{i}\right)_{i=1}^{m}$ and an integer $n \geq-\lambda_{m}$ the Young diagram $\lambda+n$ is defined as $\lambda+n=\left(\lambda_{i}+n\right)_{i=1}^{m}$.

Corollary 3 Let $M \geq N$ be positive integers, $\alpha, \gamma$ be words of length $M$ and $\beta$ a word of length $N$ such that $|\alpha|_{1}=|\gamma|_{1}, d(\gamma)-d(\alpha)=d(\beta)+|\beta|_{1}\left(|\alpha|_{0}-|\beta|_{0}\right)$ and $|\beta|_{0,|\beta|_{1}+1} \geq|\beta|_{0}-|\alpha|_{0}$. Furthermore, let 
$1^{|\beta|_{1}} 0^{|\beta|_{0}}$ be the word of length $N$ having $|\beta|_{1}|\beta|_{0}$ inversions. Then it holds

$$
\vec{r}_{\alpha, \beta}^{\gamma, 1^{|\beta|_{1}} 0^{|\beta|_{0}}}=c_{\lambda(\alpha), \lambda(\beta)+\left(|\alpha|_{0}-|\beta|_{0}\right)}^{\lambda(\gamma)} .
$$

\section{References}

F. Caselli, C. Krattenthaler, B. Lass, and P. Nadeau. On the number of fully packed loop configurations with a fixed associated matching. Elect. J. Comb., 11(2), 2004.

I. Fischer and P. Nadeau. Fully Packed Loops in a triangle: matchings, paths and puzzles. arXiv:1209.1262, 2012.

A. Knutson and T. Tao. Puzzles and (equivariant) cohomology of Grassmannians. Duke Math. J., 119(2): 221-260, 2003.

P. Nadeau. Fully Packed Loop configurations in a Triangle. Journ. of Comb. Theory, 120(8):2164-2188, November 2013a.

P. Nadeau. Fully Packed Loop configurations in a Triangle and Littlewood-Richardson coefficients. Journ. of Comb. Theory, 120(8):2137-2147, November 2013b.

J. Thapper. Refined counting of fully packed loop configurations. Séminaire Lotharingien de Combinatoire, 56:B56e:27, 2007.

R. Vakil. A geometric Littlewood-Richardson rule. arXiv:math/0302294, 2003.

D. Zeilberger. Proof of the alternating sign matrix conjecture. Elect. J. Comb., 3(2), 1996.

J.-B. Zuber. On the counting of Fully Packed Loop Configurations: Some new conjectures. Elect. J. Comb., 11(1), 2004. 